\title{
Research and Practice of Constructing "5A Model Intelligent Campus in all Aspects" in Higher Vocational Colleges under the Background of Education Informatization 2.0
}

\author{
Shuangyuan $\mathrm{Li}$ \\ Jilin Institute of Chemical Technology \\ Jilin City, Jilin Province, China \\ Email: lsy@jlict.edu.cn
}

\author{
Mingjian Li \\ School of Economics and Management \\ Jilin Institute of Chemical Technology \\ Jilin City, Jilin Province, China
}

\begin{abstract}
In order to speed up the construction of the smart campus, based on the research and practice of "unified planning, application services, digital resources, infrastructure, development of teachers and students", this paper puts forward the construction plan of "Omnibearing 5A Smart Campus" in higher vocational colleges. Under the background of the continuous advancement of information construction in colleges and universities throughout the country, the intelligent campus construction scheme proposed in this paper aims at eliminating the "Islanding" existing in universities information system, making the chaotic data available for the later construction of intelligent campus, and providing effective data basis for teachers and students work and study or daily life.
\end{abstract}

Keywords-smart campus; Big data; Vocational colleges; Education Informatization

\section{INTRODUCTION}

The new concept of "Smarter Planet" was first proposed by IBM in November 2008, and IBM's "Smarter Planet" idea was affirmed by US President Barack Obama the following January. In August of the year, IBM released the plan of "Smarter Planet wins in China", and the construction strategy of "Smarter Planet" was widely recognized around the world. Under the guidance of the concept of "Smarter Planet", the concept of "Smart Campus" came into being in China[1].

In recent years, the development trend of smart campus abroad mainly focuses on the following aspects:

- In addition to the technical communication among learners through social media, the school has built a comprehensive information service platform to achieve cross-platform social communication[2].

- Anytime and anywhere intelligent teaching management. Foreign campuses have used the Internet of things and cloud computing technology to build Smart Campus supported by cloud platform, break the information island, and realize the real-time sharing of intelligent teaching and educational information.
- $\quad$ Using big data technology for the students to make a study plan. In the future, teachers can master the panoramic view of the whole class's learning situation and make teaching plans more pertinently. The principal can understand the progress of the whole school's teaching and research work, the use of information equipment and the key weaknesses in the overall teaching through the collated data[3].

- Internet of things technology to build an intelligent campus. The Internet of Things (IOT) provides intelligent infrastructure for the smart campus, and realizes the interconnection of things through sensors to create a safe and comfortable campus environment for teachers and students.

The Education Informatization action plan2.0 officially put forward by the ministry of education on April 13, 2018, which is an upgrade of Education Informatization. Education Informatization should realize the transformation from special resources to large resources; From improving students' IT application ability to improving their IT literacy; From application convergence to innovation convergence[4].

In the construction of smart campus, some universities in China are in the forefront. For example, in "the twelfth fiveyear plan" of informatization, Zhejiang University has vigorously carried out the construction of "Smart Campus", creating ubiquitous network learning, integrated innovative network scientific research, transparent and efficient campus management, rich and colorful campus culture, and convenient and thoughtful campus life. It has achieved the goal of building an intelligent campus of "Green and energy efficient type, Peaceful and harmonious type, scientific decision making type and convenient service type". Nanjing University of Posts And Telecommunications, Southwest University, Tongji University and other universities also vigorously carry out the construction of smart campus according to their own conditions, and have made their own achievements. In terms of theoretical research, Huang Ronghuai, Shen Jie, Zhou Tong, Li Chunruo, Chen Cuizhu and other scholars have studied the connotation of smart campus from multiple perspectives, and summarized the characteristics of smart campus: comprehensive environmental 
perception, seamless network communication, massive data support, open learning environment, and personalized services for teachers and students[5].

This paper puts forward the "5A" construction plan through campus investigation, status analysis and other survey methods to speed up the informatization construction of higher vocational colleges.

\section{PERSPECTIVE ON THE CONSTRUCTION OF SMART CAMPUS IN HighER VOCATIONAL COLLEGES}

The construction of Smart Campus has gradually become the new target and trend of the development of higher vocational colleges' Education Informatization. At present, the thinking mode of Smart Campus construction in higher vocational colleges is stuck in the stage of Digital Campus construction, and the understanding of Smart Campus is not clear enough. Some people think that the already built Digital Campus can directly apply for Smart Campus, while others think that the Smart Campus can be built by adding Internet of things and sensor equipment on the basis of the Digital Campus. Such views not only confuse the concept of Digital Campus and Smart Campus, but also affect the creation of Smart Campus. It takes a long time to transition from the Digital Campus stage of most existing universities to the Smart Campus stage.

The construction of the intelligent campus in higher vocational colleges is a comprehensive system and a large platform. It emphasizes the integration of service and data information, and gives fast and comprehensive service to teachers and students to the maximum extent. In the investigation, it is found that most universities still have "Islanding", and there is no necessary connection between various systems, so there is a lack of integration. This is completely inconsistent with the concept of a smart campus.

In the investigation, it is found that the smart campus construction is not highly positioned and the design limitations are common. It mainly reflects three aspects:

- Most schools are planned, designed, deployed and communicated with commercial companies by information departments. This model helps schools open the door to Smart Campus. However, it is difficult for a company or a department to complete the complex intelligent campus construction.

- The school focuses on the construction of the basic functions of the Smart Campus, rather than the development of the smart functions of the Smart Campus.

- The school is limited to the construction of some smart functions of Smart Campus, and cannot carry out allround smart development and application.

The purpose of this thesis is to uphold the based on the "people", to "things" as the center, in order to "service" as the starting point, provide higher vocational colleges can be born, can solve the actual demand of Smart Campus innovation solutions, and first clarify Digital Campus and Smart Campus. On the basis of making full use of existing Digital Campus products of colleges and universities, integrating Internet of things, big data, cloud computing and artificial intelligence technologies, a set of feasible Smart Campus solutions are constructed, and the wisdom of smart campus is gradually enhanced[6].

The significance of the study is to solve at the beginning of the construction of higher vocational colleges as a result of the lack of unified planning and deployment of the status of each application system cannot be connected, eliminate the phenomenon of information island and make the data information for the school work, study and life to provide digital reference, the top-level design and modularization development by combining technique.

\section{OMNIBEARING 5A "SMART CAMPUS" CONSTRUCTION IDEAS}

This topic proposed construction scheme of "Omnibearing 5A Smart Campus", from "Unified planning, Application services, Digital resources, Infrastructure, Teachers and students development" five aspects, in principle, adhere to the "Unified planning, Implement step by step, Gradually improve", "Smart campus" big platform also need highly sharing and openness. In the initial stage of Smart Campus, it is difficult to achieve the function of wisdom overnight, and it needs to accumulate gradually from the level of theory and practice. It is necessary to leave space and vision for "wisdom" in the construction of Smart Campus, and guide higher vocational colleges to maximize the pursuit of campus smart on the basis of existing technologies.

\section{A. Construction goals, clear mind}

At present, due to the limited technical development level and technical introduction ability of higher vocational colleges, it is common for higher vocational colleges to locate the construction of Smart Campus in some one-sided functional phenomenon. This topic studies how to effectively achieve the top-level design, how to efficiently connect all links, so as to accelerate the construction of Smart Campus in vocational colleges.

At present, the introduction of advanced technology in higher vocational colleges is relatively lagging behind. Advanced technology is powerful support for Smart Campus. It is precisely because human technology has developed to a certain extent that the concept of Smart Campus comes into being. Generally speaking, the technologies supporting Smart Campus mainly include hardware and software technologies. At present, higher vocational colleges mainly introduce technology passively through enterprises. These massproduced products lead to the sameness of Smart Campus and lack of characteristics of each college, which is not what higher vocational colleges need. It is rare for vocational colleges to use open source software to build learning platforms that are in line with their own learning conditions. Such learning platforms should have their own learning characteristics. This paper studies the application characteristics suitable for higher vocational colleges, and seeks solutions by adopting open source software and customized development requirements. 
According to the investigation, the intelligent application urgently needed by higher vocational colleges has the following aspects, which are also important aspects to be studied in this topic:

- Microservice: mainly research and construction, microservice architecture, big data center, open integration function, full terminal experience.

- Diagnosis and improvement of higher vocational education teaching: mainly research construction, data query, decision-making consultation, various kinds of early warning, education research.

- Service center: (1) Set up the personal data center of faculty and staff: unify the entrance of staff and staff information data filling, and provide a series of services such as data filling, approval, data sharing and data reporting for different types of staff and staff. (2) Construction of student work services: wisdom welcome, wisdom learning and work, leaving school management three aspects[7].

\section{B. Research direction, data statistics}

Research idea, composition and functions of the current digital campus products classification, statistical analysis of the market share screening each classification under the mainstream products, organic combination, as Smart Campus plan, to study and analysis of each package, specify the feasible solution, can be born of Smart Campus innovation, as shown in figure 1 Smart Campus building Omnibearing 5A model.

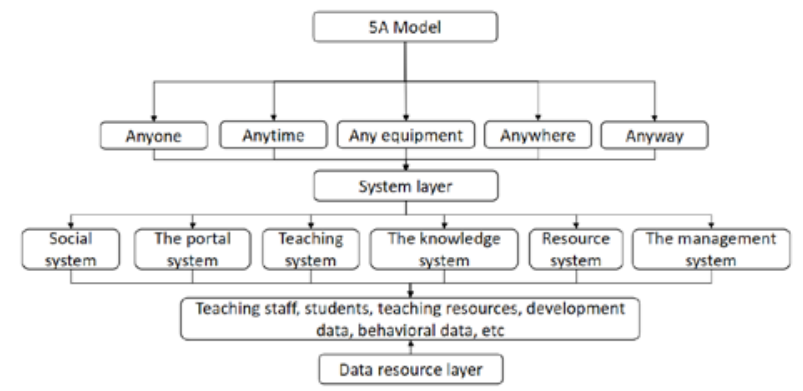

Fig. 1. 5A Model

The research method adopts the method of questionnaire survey, the literature investigation method and the method of analysis of cases. Through the method of analysis of cases, this paper makes an analysis of the service case of "One Network Operation" in Shen Zhen Polytechnic. Through the questionnaire survey of Higher Vocational colleges, the actual situation is investigated. Collecting the Thought of Intelligent Campus Construction Scheme by the literature investigation method. The project is committed to adding impetus to the realization of "the thirteenth five-year plan" information development goals.

The implementation of this construction plan is inseparable from the support of big data, and more importantly, it needs a professional team integrating information resources within the school to support the construction of Smart Campus. Since the final system is user-oriented, it is necessary to integrate all feedback problems and propose solutions in the early stage of system development, which is very critical for the information team. Therefore, the introduction and training of information quality talents is also an indispensable part of the construction of Smart Campus[8].

\section{INNOVATIVE APPLICATIONS AND KEY ISSUES}

Subject innovation, make full use of vocational colleges Digital Campus construction infrastructure, based on the present. At the technical level, it integrates advanced technologies such as cloud computing, big data, Internet of things, and artificial intelligence, with a bold outlook Innovation research mainly has the following three aspects.

- Construction of microservice: the premise is to solve problems such as data islanding, multiple identity management, application mountain, multiple application access interface, integration of various heterogeneous data sources, etc. The micro services in the system independently solve various tasks, but the microservice is connected with each other, which conforms to the concept of "Smart Campus".

- Construction of diagnosis and improvement of higher vocational education teaching: it is based on the collection and filling technology of campus big data, combined with the quality assessment index of higher vocational colleges, and comprehensively analyze teaching and other aspects on the basis of the application of big data, so as to provide leaders with reference for the continuous improvement decision of school talent cultivation.

- Construction of service center: to serve personalized user needs, and to provide universities with a one-stop comprehensive data service platform integrating information standards and specifications, data collection, data storage, data governance, decision analysis, comprehensive reports, data mining and data service interface based on big data analysis[9].

At present, the key problem is the combination of top-level design application and modular development, which is particularly prominent in the informatization construction of higher vocational colleges. First of all, we should get rid of the limitation of uniform design of information department and reduce the awkward situation of inconsistent supply and demand in the design process. By changing the design ideas, functional departments should think deeply about the application needs of their own departments, communicate with developers in depth, and translate the application needs into intelligent operation of Smart Campus scientifically and reasonably. In module design, it is not to let each department run their own affairs and get rid of the top-level design of the information department, but to strengthen the unified standard and interface design of the information department, carry out unified and coordinated deployment, guide all departments to jointly develop Smart Campus functions, participate in data mining, and seek wisdom from complex data [10]. 


\section{CONCLUSION}

In the context of the rapid development of information technology in the new era, as the country attaches more importance to the construction of Smart Campus, Chinese colleges and universities have responded positively, gradually improving the construction plan and research direction of each college from theory to practice. The construction of Smart Campus has become a necessary goal for the transformation of universities in China. In the saturation stage of Digital Campus, we should further improve the teaching quality and user experience, and promote the modern education reform. This paper puts forward the construction plan of "Omnibearing 5A Smart Campus". From the perspective of the informatization construction of colleges and universities, this paper analyzes the current transformation problems faced by higher vocational colleges, puts forward powerful solutions, and gives the specific steps at the initial stage of construction and future research direction and planning.

\section{REFERENCES}

[1] Ying Zhang. Exploration and practice of "one-stop" service model in universities under the background of big data [J]. Computer application and software, 2019,(07):128-132. ( In Chinese)
[2] Ling Li. The advanced research of colleges and universities from digital campus to intelligent campus [J]. Heilongjiang Science, 2019, (13):9293. ( In Chinese)

[3] Guoqiang Liu Jie Lin. Research on the framework design and application of smart campus construction of application-oriented undergraduate universities in the era of Internet $+[\mathrm{J}]$ Modernization of education2019(46):145-147. ( In Chinese)

[4] Hua Tan,Lijing Ma. Research on intelligent campus construction based on Internet of things and big data [J] Wireless interconnection technology2019, (11):25-27. ( In Chinese)

[5] Leixiao Hu. Exploration on the construction direction of smart campus in the era of Education Informatization 2.0[J]. China Educational Technology \& Equipment2019, (03):61-61+71. ( In Chinese)

[6] Yingxia Yang. Transformation of Education Informatization: research on construction of "Internet + smart campus"[J]. Journal of Educational Institute of Jilin Province, 2019(07):115-118. ( In Chinese)

[7] Xutao Xuan. Analysis on the construction of intelligent campus informatization in universities under the background of big data [J]. China Management Informationization, 2019(12):145-146. ( In Chinese)

[8] Baomi Li. Discussion on intelligent campus construction based on Internet of things [J]. Information \& Communications, 2019(06):170172. ( In Chinese)

[9] Peibo Sun, Lijiang Zhou. Research on the construction strategy of smart campus big data exchange center [J].Education Modernization, 2018,(50):354-355. ( In Chinese)

[10] Ying Wang. Based on "artificial intelligence + big data" intelligent campus virtual campus card innovation and practice [J]. Research on Transmission Competence, 2018(31):238. ( In Chinese) 